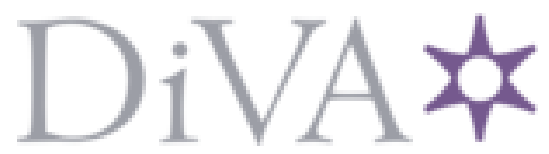

http://www.diva-portal.org

This is the published version of a paper published in IEEE Transactions on Power Delivery.

Citation for the original published paper (version of record):

Almas, M S., Vanfretti, L. (2015)

RT-HIL Implementation of Hybrid Synchrophasor and GOOSE-based Passive Islanding Schemes.

IEEE Transactions on Power Delivery

http://dx.doi.org/10.1109/TPWRD.2015.2473669

Access to the published version may require subscription.

N.B. When citing this work, cite the original published paper.

Permanent link to this version:

http://urn.kb.se/resolve?urn=urn:nbn:se:kth:diva-185004 


\title{
RT-HIL Implementation of Hybrid Synchrophasor and GOOSE-based Passive Islanding Schemes
}

\author{
M. S. Almas, Student Member, IEEE, and L. Vanfretti, Senior Member, IEEE
}

\begin{abstract}
Real Time Hardware-in-the-Loop (RT-HIL) performance assessment of three different passive islanding detection methods for both local and wide-area synchrophasor measurements is carried out in this article. Islanding detection algorithms are deployed within the Phasor Measurement Unit (PMU) using logic equations. Tripping decisions are based on local and widearea synchrophasors as computed by the PMU and trips are generated using IEC 61850-8-1 Generic Object Oriented Substation Event (GOOSE) messages. The performance assessment compares these islanding detection schemes for Non Detection Zone and operation speed under different operating conditions. The test-bench demonstrated is useful for a myriad of applications in which simulation exercises in power system CAD software provides no realistic insight into the practical design and implementation challenges. Finally different communication latencies introduced due to the utilization of synchrophasors and IEC 618508-1 GOOSE messages are determined.
\end{abstract}

Index Terms-- Real-Time Hardware-in-the-Loop Simulation, PMU, Power System Islanding, Protection Relays, Synchrophasors.

\section{INTRODUCTION}

Islanding is a condition, in which, a part of the power system consisting of both loads and generation becomes isolated from the rest of the power grid, and generators continue to energize the isolated network [1]. Two types of islanding occur in a power system: intentional islanding and unintentional islanding. Intentional islanding is performed for either maintenance or load shedding purposes to protect the rest of the power grid and avoid a blackout. The isolated generators operate in both voltage and frequency control mode to provide constant voltage to local loads in the isolated network while maintaining the isolated grid frequency. Unintentional islanding occurs due to equipment failure or severe faults resulting in the opening of circuit breakers interconnecting the island with the rest of the power system. Unintentional islanding may result in hazards in power system operation and may lead to safety risks for maintenance staff. In addition, during unintentional islanding, the isolated network suffers from significant voltage and frequency variations that can damage both loads and generators within the island. Furthermore, auto-reclosing of the tie-

This work was supported in part by Nordic Energy Research through the STRONg2rid project and by Statnett SF, the Norwegian TSO.

M. S. Almas, and L. Vanfretti are with KTH Royal Institute of Technology, Stockholm, Sweden. (e-mail: \{msalmas, luigiv\}@kth.se)

L. Vanfretti, is with Statnett SF, Research and Development, Oslo, Norway (email: luigi.vanfretti@statnett.no) line, which is a standard automated procedure followed in case of temporary faults, results in out-of-phase and unsynchronized reclosing when the system is subject to unintentional islanding.

\section{A. Paper Motivation}

For the particular case of Distributed Generation (DG), the IEEE Standard for Interconnecting Distributed Resources with Electric Power Systems (IEEE Std. 1547-2008) [2] states that the DG must be disconnected from the isolated grid within 2 seconds after an unintentional islanding event. This maximum delay of 2 seconds includes islanding detection, trip signal generation, trip signal transfer and breaker opening for the connected DG. It is therefore important to, not only have fast islanding detection algorithms, but also to have low-latency trip signal transfer schemes to open the breaker.

Synchrophasors from multiple local and remote measurement locations in the grid may be exploited for islanding detection [3]. A hybrid synchrophasor and IEC 61850-8-1 (GOOSE) [4]-based scheme can provide faster operation times as compared to traditional hardwired schemes, as it omits the output circuitry delay, e.g. "make or break" delay of auxiliary signaling relays which is typically $8-10 \mathrm{~ms}$ [5]. Even with digital protection relays having opto-isolated digital I/Os, the utilization of GOOSE message results in tripping time 3-6 ms faster than the hardwired digital I/O based trippings [6].

\section{B. Literature Review}

It has been reported in [7] that synchrophasors based islanding detection schemes can provide fast, reliable and accurate detection of islanding condition under different power system operating conditions. However, these studies were based on off-line simulations and actual data from real hardware PMUs were not used for performance analysis of the proposed islanding detection algorithms. Thus, the communication latencies introduced due to the utilization of synchrophasor measurements were not taken into account. In [8], wide-area synchrophasor measurements are utilized to continuously monitor the phase of generators to determine synchronism within the generators and loss of synchronism is interpreted as loss-of-mains scenario. This algorithm was tested using archived synchrophasor measurements from the power grid, and the prototype was deployed in Labview on a non-Real-Time Operating System. The different communication latencies associated with this algorithm were not taken into account. In [9], a synchrophasor based islanding detection scheme is implemented as an add-on feature in a software version of a phasor data concentrator (PDC). PDC software is typically installed on servers with Windows OS which is not a Real-Time Operating System 
(RTOS). The software PDCs cannot be time synchronized with sufficient accuracy in such environment and therefore PDC processing delay cannot be thoroughly evaluated. The processing time of the PDC is dependent on its configured waiting time, which refers to the maximum amount of time to wait for all inputs to be received, time-aligned and concentrated in a specific output stream. This waiting period is generally 100-200 ms (depending upon the geographical location of the PMUs). This adds extra delay to the overall operating time of a synchrophasor-based islanding detection scheme and thus they may violate the anti-islanding criteria specified in IEEE Std. 1547-2008 [2]. In order to accurately identify the communication delays incurred by the PDCs, a hard real-time operating system for PDCs is essential. In addition, current PDCs have been designed for wide-area monitoring purposes, and therefore, face challenges to meet real-time requirements for time-critical applications (e.g. protection).

\section{Paper Contribution}

This paper presents the implementation and RT-HIL performance assessment of a hybrid synchrophasor and IEC 61850-8-1 (GOOSE) [4] -based passive islanding detection algorithms utilizing both local and wide-area synchrophasors. Real-time hardware-in-the-loop (RT-HIL) simulation [11] [12] including PMUs from Schweitzer Engineering Laboratories [13] is executed for performance analysis of these schemes. Methods to accurately calculate different latencies associated with synchrophasor-based islanding schemes such as PMU filtering delay, PMU synchrophasor computation delay, PMU algorithm execution delay, PMU time-alignment delay (for remote measurements), synchrophasor frame formation delay and GOOSE latencies are also presented.

Different islanding detection algorithms are deployed as simple logic equations within the PMU. This approach is generic, as logic equations are supported by all the microprocessor-based protection relays. Performance assessment of the proposed algorithms is performed by evaluating the criteria documented in IEEE Std. 1547-2008 [2]. The Non Detection Zone (NDZ) [14] is evaluated for both active and reactive power mismatches, between generation and local load, for all algorithms.

The proposed approach is subjected to the minimum possible communication latency and can be used for fast prototyping of any passive islanding detection algorithm that utilizes local or wide-area synchrophasor measurements.

\section{ISLANDING DETECTION METHODOLOGIES}

This section gives a brief overview on different islanding detection methods commonly used by utilities.

\section{A. Passive Islanding Detection Methods}

These methods are based only on the electrical quantities being monitored. These methods detect an islanding condition when these electrical quantities violate a pre-specified threshold.

Passive islanding detection methods can be implemented in two ways

- Local-based Passive Islanding Detection: These are based on local measurements at the DG side. However they have a large non-detection zone (NDZ) [14], which is defined as the range of power mismatch between DG supply and local load for which the particular islanding detection method may fail.

- Wide Area Passive Islanding Detection: If the power mismatch between the DG and the local load is negligible, the local-based passive islanding detection methods may fail. Wide area-based passive islanding detection schemes utilize synchrophasors from both the DG and utility side to detect an islanding condition [7].

These methods require intensive offline simulations to set the threshold limit to accurately identify islanding conditions, as the performance of these schemes depends on the protection relay settings. Too rigorous limits can result in false islanding detection and tripping of the DG in normal operating conditions while too loose settings will result in longer operation time and therefore violate of $\mathrm{DG}$ disconnection requirement of $2 \mathrm{~s}$ as specified by IEEE Std. 1547-2008 [2].

\section{B. Active Islanding Detection Methods}

In active islanding detection methods, a small perturbation is introduced in the system deliberately. These methods have a very small NDZ and can detect islanding conditions even if there is a perfect match between DG and local load [15][16].

Active islanding detection schemes are relatively slow in detecting islands as compared to passive islanding methods. This is because they rely on the response of the injected perturbation in the system which takes additional time to detect.

\section{Power System Test CASE Modeling}

A modified IEEE 3-machine 9-bus system [10] is modeled in MATLAB/Simulink for real-time execution and is shown in Fig. 1. The system contains 3 generators, 9 buses and 3 loads. The system was modified for $50 \mathrm{~Hz}$ nominal operating frequency and real-time simulation purposes (including detailed three phase branch/breaker modeling). If CB-1a, CB-1b and CB-2a, CB-2b are opened simultaneously, this results in an islanding condition with G1 supplying power to the Load A at Bus 5. Once the breakers are opened and the island is formed, this condition needs to be detected and the DG (in this case G1) needs to be disconnected from the isolated network within 2 seconds as specified by IEEE Std. 1547-2008 [2]. A PMU from Schweitzer Engineering Laboratories SEL-421 [13] is interfaced at Bus-4 (DG side).

\section{EXPERIMENTAL SETUP}

The real-time hardware-in-the-loop (RT-HIL) experimental setup is shown in Fig. 2 and was configured at SmarTS-Lab [17]. The power system model is executed in real-time using Opal-RT's eMEGAsim Real-Time Simulator (RTS) [11]. The three phase voltage and currents of Bus-4 are accessed through the analog outputs of the RTS. These low-level analog signals are amplified to nominal range of 300 Volts and 1 Ampere using linear amplifiers. Amplified analog voltage and current signals are fed to the PMU which computes phasors for all the phases and positive sequence for both voltage and current that are reported at 50 frames/s. 


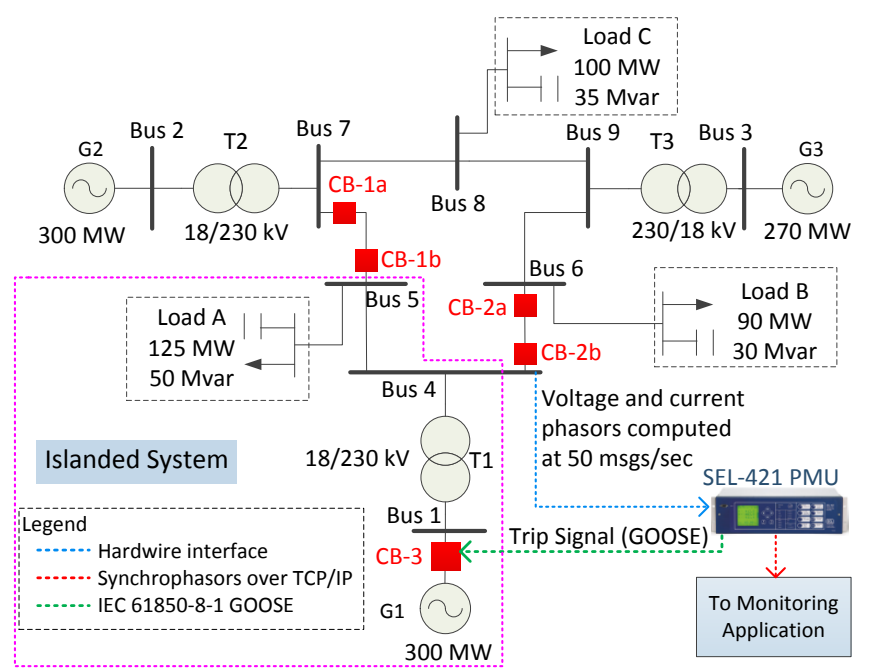

Fig. 1. IEEE 3-machine, 9-bus power system modelled in MATLAB/Simulink. The islanded region is outlined.

The synchrophasors are internally utilized by the PMU to execute the islanding detection algorithms deployed using logic equations. These logic equations are discussed in the next section. Once the islanding condition is detected, a trip command is generated by the PMU and a GOOSE message with changed status is sent to the RTS. This GOOSE message published by the PMU has a subscription from the RTS that is configured to open circuit breaker CB-3 in the model. This disconnects the DG (G1) from the isolated network. The performance evaluation of islanding detection algorithm is carried out by calculating the time difference between the opening of CB-1 and CB-2 to form an island and the tripping of CB-3 caused by the PMU to disconnect G1. The non-detection zone (NDZ) [14] is determined by changing both the active and reactive power consumption of Load A to simulate different operating conditions.

The RTS' GOOSE subscription is configured through a GOOSE subscriber. This is achieved through a block that requires a IED Capability Description (ICD) file. A ICD file describes the complete capability of an IED. In order to subscribe to a GOOSE message the Multi-cast address of publication as well as its identifier (AppId) are used to produce control signals corresponding to the GOOSE message received through the IEC 61850 network [4].

In order to monitor the synchrophasors and to analyze the behavior of the islanding detection algorithms, a simple monitoring application was developed in LabView. The PMU was configured to stream out all computed phasors. Important states of the islanding detection algorithm's and the tripping signal were configured as digital output signals within the PMU stream as specified by IEEE Standard for Synchrophasor data transfer for Power Systems (IEEE Std. C37.118.2-2011) [18]. This PMU stream is received in a workstation using Statnett's Synchrophasor Software Development Kit (S3DK) [19] which provide real-time synchrophasor data in the LabView environment. Within LabView, these raw measurements are presented in real-time displays for monitoring purposes and are stored for further analysis.

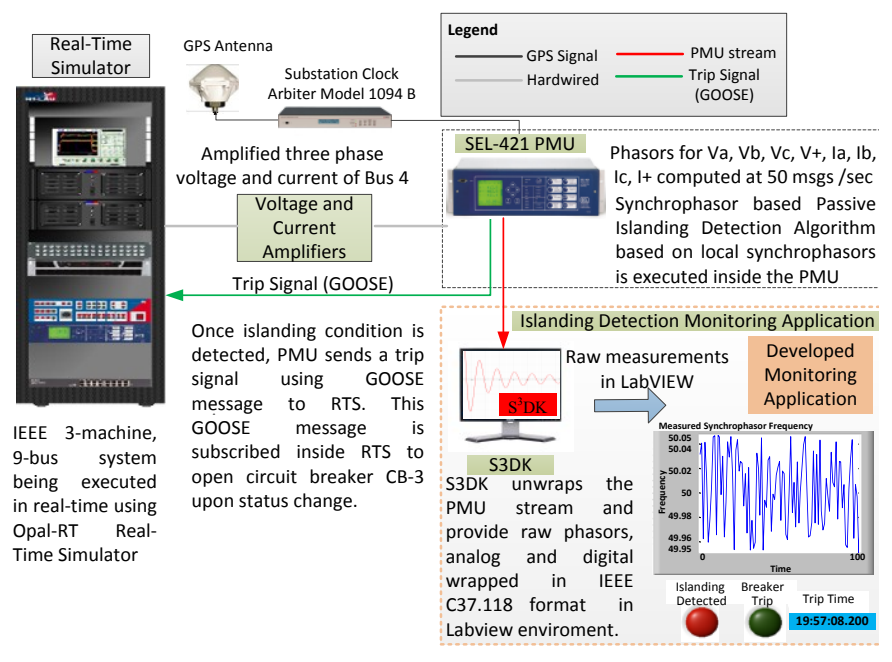

Fig. 2. Experimental setup for performance analysis of synchcrophasor based islanding detection schemes using local synchrophasors

\section{IMPLEMENTATION OF LOCAL PASSIVE ISLANDING SCHEMES}

This section gives a detail of different passive islanding detection methods implemented in the PMU. Using logic equations, it is possible to deploy a variety of passive islanding detection schemes such as those presented in Section II-A. However, in this section, only passive islanding detection schemes that are computationally efficient and that use local synchrophasor measurements were implemented. The description of each islanding detection method and its implementation using logic equations is presented.

\section{A. Over/Under Voltage}

When an island is formed, the voltage magnitude at DG side changes significantly if there is a large variation in DG power supply and the connected local load. If this voltage magnitude variation persists for a specific period of time (10 cycles), an island condition is detected and a trip signal is generated [20]. The 10 cycle delay incorporated in these schemes is to accommodate the instantaneous tripping of the protection function by the corresponding protection relay and the opening time of the breaker. Instantaneous protection operating time varies between 10-40 ms depending upon the type of protection, the fastest being instantaneous overcurrent $(10-15 \mathrm{~ms})$, followed by distance protection (15-25 ms) and differential protection $(20-40 \mathrm{~ms})$. In addition to this instantaneous protection operation time, the opening time of $230 \mathrm{kV}$ circuit breaker is considered, which is between $3-5$ cycles $(60-100$ $\mathrm{ms})$. As a fair estimate, the timer of 10 cycles $(200 \mathrm{~ms})$ is used to accommodate for all these transients in the power system. Figure 3 shows the logic diagram of this deployed islanding method and its respective logic equation programmed in the PMU.

Once the local voltage phasor magnitude exceeds $1.1 \mathrm{pu}$ or goes below $0.9 \mathrm{pu}$, a conditioning timer PCT01 activates. If the voltage violates this threshold for 10 cycles, the output of the timer (PCT01Q) changes its status from 0 to 1 . This output is configured to generate the general trip signal. This general trip is also published as a IEC 61850-8-1 GOOSE message. 
Figure 4 shows synchrophasor positive sequence voltage magnitude as computed by the PMU and the response of the over/under voltage based islanding detection method when there is a $30 \%$ reactive power mismatch between G1 and Load A. At $\mathrm{t}=0.74 \mathrm{~s}$, the circuit breakers CB-1 and CB-2 open resulting in an island. The synchrophasor voltage (Fig. 4) starts increasing and at $\mathrm{t}=1.74 \mathrm{~s}$, the synchrophasor voltage goes above 1.1 pu resulting in the change in status of digital variable PSV 50 and starts the timer PCT 01. Once the timer reaches 10 cycles and the over-voltage condition is sustained, the timer PCT 01 changes the status of its output PSV 52 at $\mathrm{t}=$ $1.94 \mathrm{~s}$. This output of the over-voltage timer is published as a GOOSE message that opens the circuit breaker CB-3 at $\mathrm{t}=$ $1.98 \mathrm{~s}$ to disconnect the DG from the isolated island. The total operating time for anti-islanding scheme is the difference between time at which island is formed (i.e. opening time of CB$1_{a, b}$ and CB- $2_{a, b}$, which in this case is $t=0.74 \mathrm{~s}$, and the opening of CB-3 due to over-voltage condition at $\mathrm{t}=1.98 \mathrm{~s}$. Thus total operation time for this scheme with $30 \%$ reactive power mismatch is $1.24 \mathrm{~s}$.

The Non Detection Zone (NDZ) is calculated by fixing both the active and reactive power output of generator "G1" and changing the active and reactive power consumption of "Load A". The NDZ for the over/under voltage based islanding detection method is shown in Fig. 5a. The scheme results in successful islanding detection only if there is a significant active power or reactive power mismatch between the DG and the local load in the island. This scheme requires a reactive power mismatch of at least $20 \%$ or active power mismatch of over 30 $\%$.

\section{B. Over/Under Frequency}

The frequency of a power system reflects active power mismatches between generation and consumption. During normal operation, the power system is interconnected and the grid frequency varies within $\pm 0.5-1 \%( \pm 0.25-0.50 \mathrm{~Hz}$ for 50 $\mathrm{Hz}$ system) of the nominal frequency. However, in the case of islanding, the power mismatch between isolated DG and local load causes the frequency to rise (over frequency) or drop below (under frequency) the allowed thresholds. This physical behavior is used to set the trip command that isolates the DG
[21]. PMUs estimate frequency deviation and rate-of-change of frequency from the positive-sequence synchrophasor voltage angle. The frequency deviation is calculated as;

$$
f_{k}=\frac{\left(\theta_{k}-\theta_{k-1}\right)}{\Delta t \times 360}=\frac{\left(\theta_{k}-\theta_{k-1}\right)}{\left(1 / F_{s}\right) \times 360}
$$

where $\theta_{k}$ and $\theta_{k-1}$ are consecutive positive-sequence synchrophasor voltage angles computed at $k$ and $k-1 . \Delta t$ is the time difference between the angle calculations. $F_{s}$ is the synchrophasor reporting rate of the PMU which in this study is 50 frames/s.

Figure 6a shows the logic diagram of the over/under synchrophasor frequency based passive islanding detection algorithm and its respective logic equation programmed in the PMU. The over frequency threshold was set to $51 \mathrm{~Hz}$ and the under frequency was set to $49 \mathrm{~Hz}$ [21]. The NDZ for the over/under frequency based islanding detection method is shown in Fig. 5b. NDZ is much smaller as compared to over/under voltage based islanding scheme (Fig. 5a). However, it still requires an active or reactive power mismatch of at least $10 \%$ to accurately detect the islanding condition.

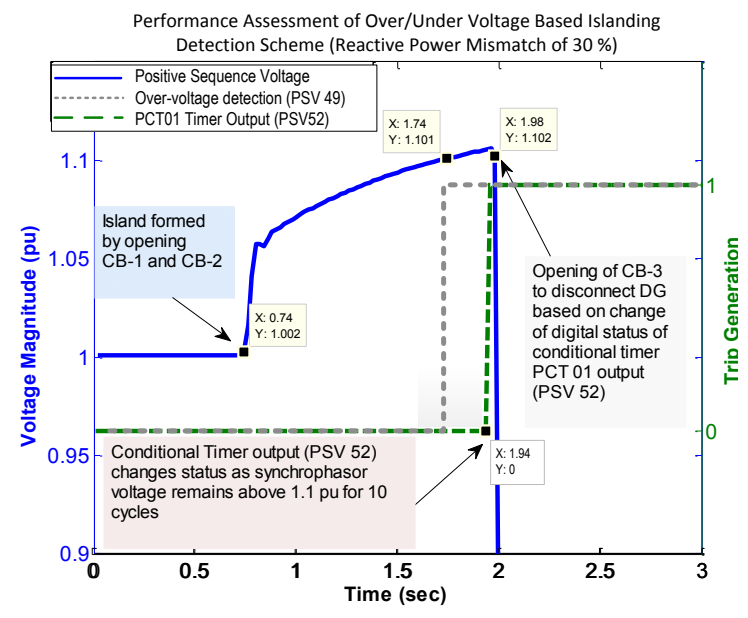

Fig.4. Over/under voltage based passive islanding detection scheme for $30 \%$ reactive power mismatch. The total operating time is $1.24 \mathrm{~s}$.

PMV53 := V1YPMM \% Storing positive sequence voltage phasor magnitude in user defined
Pnalog
PMV54 := $1.100000 \%$ Storing upper threshold value of 1.1 in user defined analog
PMV55 := $0.900000 \%$ Storing lower threshold value of 0.9 in user defined analog
than upper threshold
PSV02 := PMV53 < PMV55 \% SET if positive sequence voltage phasor magnitude is lesser than
lower threshold
PSV03:= PSV01 OR PSV02 \% Logical OR operation. SET if either upper or lower threshold is
exceeded.

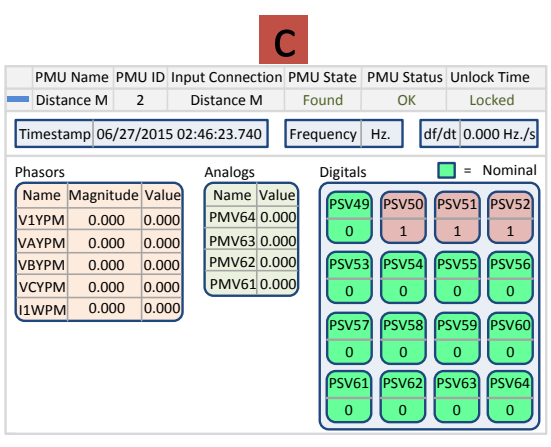

Configured Phasors, Analogs and Digitals for the PMU. These values are packgaed in C37.118.2 frame and are streamed out as synchrophasors

Fig.3. (a) Logic diagram (b) Protection logic equations used to deploy the algorithm within the PMU. (c) Synchrophasor frame showing the configured phasors, analogs and digitals. PSV 49-PSV 52 stores the digital status as configured by the protection logic equations (b). 


\section{Rate of Change of Frequency (ROCOF)}

The Rate of Change of Frequency (ROCOF) method is frequently used to deploy Loss of Main (LOM) detection because of its simplicity and cost effectiveness as compared to other methods [22]. When the island is formed, the active power imbalance between the DG and the local load results in a dynamic change in frequency.

PMUs are capable of calculating ROCOF and it is streamed out in the synchrophasor frame according to IEEE Std. C37.118.2-2011 [18]. PMUs calculate the ROCOF by computing time derivative of the difference in consecutive frequency estimations according to the following equation

$$
\text { ROCOF }=\frac{d f}{d t}=\frac{f_{k}-f_{k-1}}{\left(1 / F_{s}\right)}
$$

where $f_{k}$ and $f_{k-1}$ are consecutive frequencies estimated by the PMU at time $k$ and $k-1 . F_{s}$ is the synchrophasor reporting rate of the PMU which in this study is 50 frames/s.

ROCOF can be readily used to implement islanding detection. In this study, the implementation of the ROCOF threshold limit is set to $0.2 \mathrm{~Hz} / \mathrm{s}$ to account for islanding. The logic diagram and the respective logic equations for synchrophasor ROCOF based islanding detection algorithm are shown in Fig. $6 b$.

The NDZ for the ROCOF-based islanding detection method is shown in Fig. 5c. The NDZ is similar to that of the over/under frequency-based islanding scheme (Fig. 5b). However, it still requires an active or reactive power mismatch of at least $10 \%$ to accurately detect the islanding condition.

\section{RT-HIL SimULATION RESUlTS FOR LOCAL PASSIVE ISLANDING SCHEMES}

Passive islanding detection schemes exploiting local synchrophasors are computationally efficient and cost effective. Trip decisions depend on these local measurements; and therefore, communication delays associated with remote measurements have a minimum impact on their performance.

The comparison of the operation time of the implemented schemes for different active power and reactive power mismatch is shown in Fig. 7 and 8 respectively. These operation times include the islanding detection algorithm processing time, PMU phasor computation time, GOOSE message communication delay and circuit breaker opening time. The over/under voltage based islanding detection scheme shows a faster operation time with an increase in active and reactive power mismatch. The reactive power mismatch shows faster operation time as compared to active power mismatch for voltage based islanding detection schemes.

By taking into consideration all the PMU processing delays and communication delays in the transmission of the GOOSE message, it can be noted that over/under voltage based islanding detection schemes fulfil the requirement of $2 \mathrm{~s}$ for DG disconnection if the reactive power mismatch between DG and local load is larger than $20 \%$ (Fig. 8).

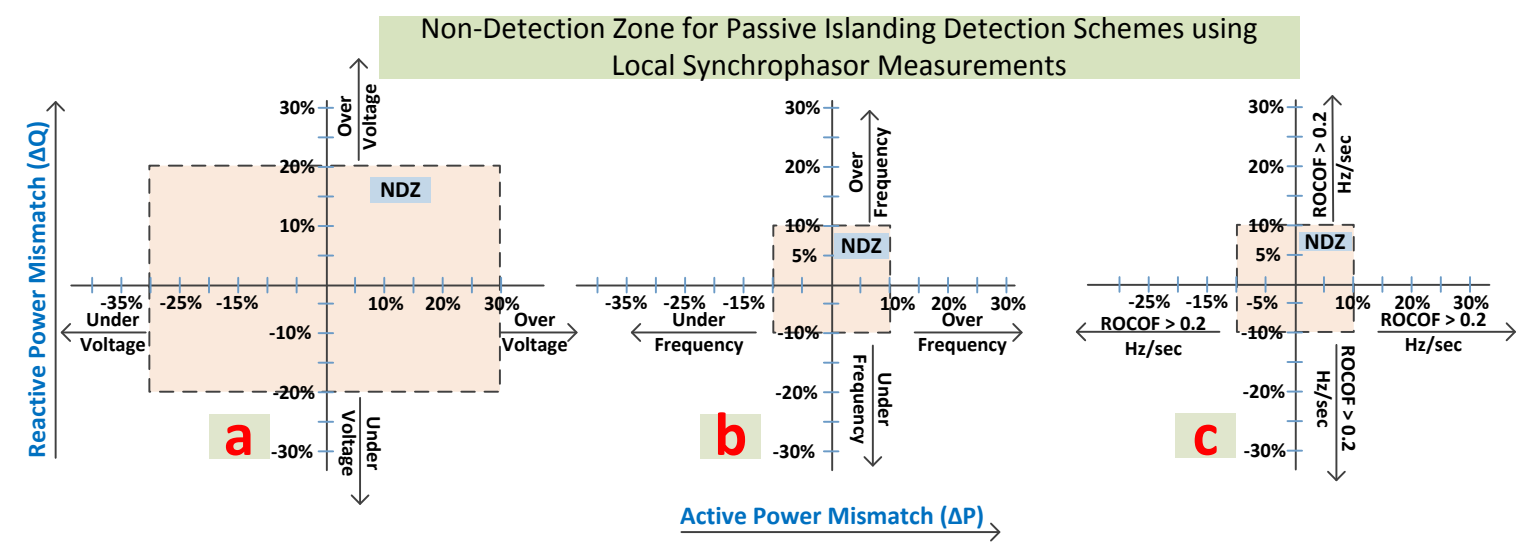

Fig.5. Non Detection Zone (NDZ) for (a) Under/Over Voltage based islanding detection scheme with $\mathrm{V}_{\min }=0.9 \mathrm{pu}$, $\mathrm{V}_{\max }=1.1 \mathrm{pu}$, (b) Under/Over Frequency based detection method with $\mathrm{f}_{\min }=49 \mathrm{~Hz}$ and $\mathrm{f}_{\max }=51 \mathrm{~Hz}$, and (c) ROCOF based islanding detection scheme with ROCOF threshold limit set to $0.2 \mathrm{~Hz} / \mathrm{s}$

Synchrophasor
Frequency
PMV53:= FREQPM \% Storing measured synchrophasor frequency in
user defined analog
PMV54 := 51.00000 \% Storing upper threshold value of $51 \mathrm{~Hz}$ in user
defined analog
PMV55 := 49.00000 \% Storing lower threshold value of $49 \mathrm{~Hz}$ in user
defined analog
PSV01 := (PMV53 > PMV54) OR (PMV53 < PMV55) \% SET if measured
synchrophasor frequency
Logic equations for PCT01 (Timer) and Synchrophasors digitals are
the same as in Fig. 3(b)

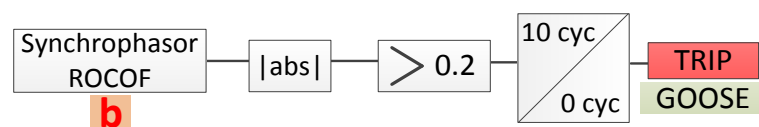

PMV53 := DFDTPM \% Storing measured synchrophasor ROCOF in user defined analog

PMV54 := $0.2 \%$ Storing threshold value of $0.2 \mathrm{~Hz} / \mathrm{sec}$ in user defined analog

PSV01 := Abs(PMV53) > PMV54 \% SET if Absolute value of measured synchrophasor ROCOF is greater than $0.2 \mathrm{~Hz} / \mathrm{sec}$.

Logic equations for PCT01 (Timer) and Synchrophasors digitals are the same as in Fig. $3(\mathrm{~b})$

Fig.6. (a) Logic diagram and protection logic equations used to deploy the over/under synchrophasor frequency based islanding detection algorithm within the PMU (b) Logic diagram and protection logic equations used to deploy the synchrophasor ROCOF based islanding detection 


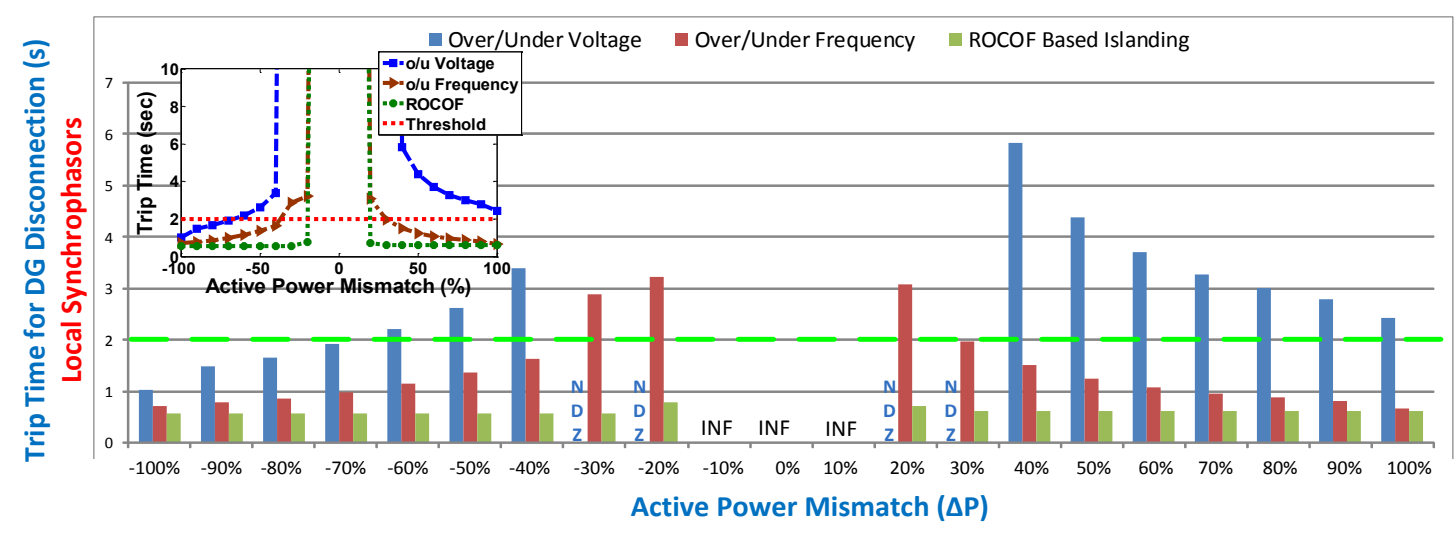

Fig.7. Comparison of operation time of passive islanding detection schemes (local synchrophasors) when there is an active power mismatch.

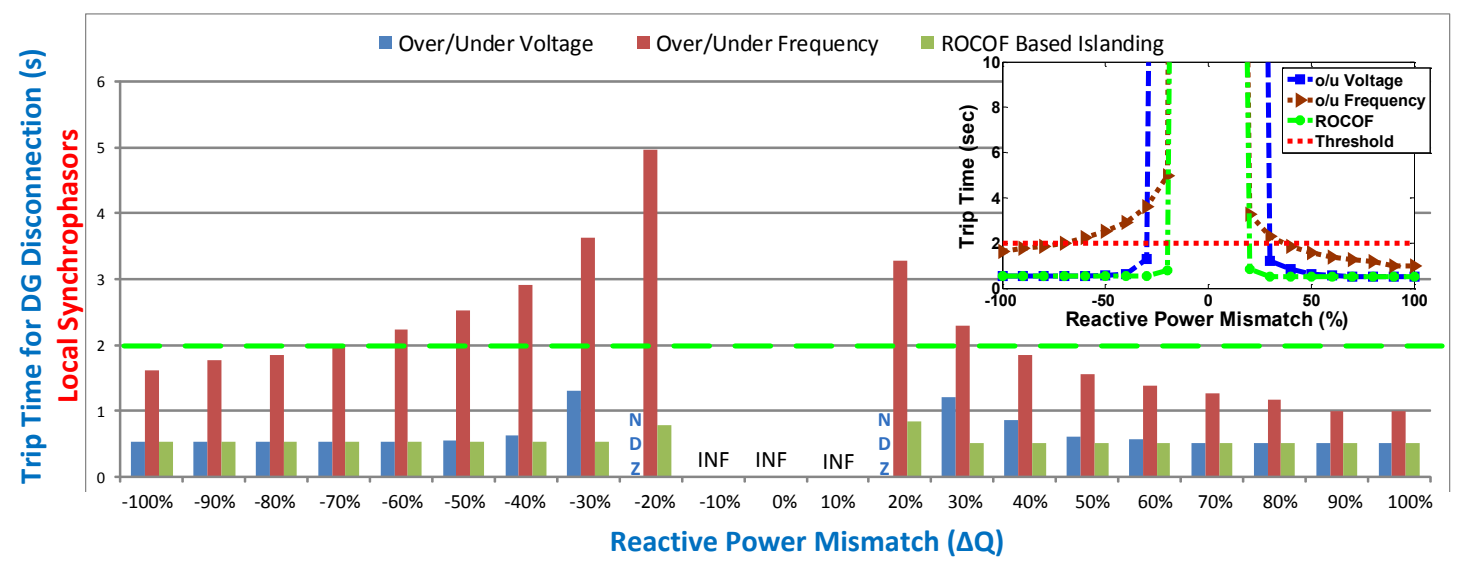

Fig.8. Comparison of operation time of passive islanding detection schemes (local synchrophasors) when there is reactive power mismatch.

Over/under frequency based islanding detection methods have a wider islanding detection zone (Fig. 5b). However, acceptable operation requires an active power mismatch between DG and the local load larger than $30 \%$ (Fig. 7). Over/under frequency based islanding detection schemes perform very slowly if there is only a reactive power mismatch (Fig. 8). ROCOF based islanding detection schemes are highly reliable if the power mismatch between DG and local load is more than $10 \%$. ROCOF results in a faster operation than the rest of the methods discussed in this study for both active and reactive power mismatch. However, even ROCOF does not detect the islanding condition if the power mismatch between DG and the local loads is less than 10\% (Fig. 7 and Fig. 8).

The accuracy of the passive islanding detection schemes depends on the accuracy of the synchrophasor being computed by the PMU. Transients have a major effect on both estimated frequency and ROCOF. In [23] and [24], the authors have carried out extensive experiments to carry out both steady-state and dynamic compliance testing for PMUs from three different vendors. Most of the commercial PMUs used in this study failed the dynamic compliance testing, however, once the dynamic (transient) condition is over, all the three PMUs remain within the maximum allowable tolerance limits for frequency error and ROCOF thresholds. The authors believe that the 10 cycle timer used in the simulation accommodates for transients (fault + breaker opening), and therefore, the PMUs frequency estimation can be considered reliable enough during the post transient disturbance condition.

All the local synchrophasor-based islanding detection techniques discussed in Section-V are, in reality, performed by stand-alone protection relays e.g. over/under voltage protection relay (ANSI Code 59/27), over/under frequency (ANSI Code 81) and ROCOF (ANSI Code 81R). All these protection relays are configured either to provide instantaneous tripping or definite time based tripping (once the fault is picked-up, a relay waits for a certain pre-configured time before issuing a trip). These protection relays won't require synchrophasor estimation and subsequent utilization to perform islanding.

Local synchrophasor-based islanding detection schemes are the first logical step towards the implementation of more complex and wide-area synchrophasor-based islanding schemes. This helps increasing the confidence of Transmission System Operators (TSOs) and Distribution System Operators (DSOs) on PMU technology utilization in time-critical protection schemes such as anti-islanding. As a result, this will build trust on utilizing wide-area synchrophasor technology for different protection schemes that may benefit from it.

The islanding detection schemes presented and tested in Section-V can be utilized for both transmission and distribution systems. As the schemes are based on synchrophasors, limitation of the current PMU technology (their accuracy and compliance with the standard), make them suitable to be utilized only in transmission networks. However with the ongoing development of more accurate and robust PMUs for 
distribution systems, the same schemes can be used in distribution grid [8] [25] in the future.

The proposed hybrid synchrophasor and IEC 61850-8-1based islanding scheme involves some unavoidable time delays. These delays are due to the use of PMUs and the communication link involved for streaming out synchrophasors and GOOSE messages. Figure 9 depicts the calculation of different latencies. They are explained below.

PMU Processing Delay: Synchrophasors are computed by filtering the incoming analog voltage and current signals fed to the PMU's Voltage Transformer (VT) and Current Transformer (CT) transducers respectively. These filtered voltage and current measurements are used to compute synchrophasors. In order to accurately identify delays, event recordings of the PMU were retrieved and the raw input analog signal, filtered measurements in the PMU and the computed synchrophasor of PMU were plotted. The difference between raw input analog signal from a test-set and the filtered measurements retrieved by PMU gives the filter delay which is $30 \mathrm{~ms}$ (Fig. 9a). The difference between filtered measurements and the associated synchrophasor provides synchrophasor algorithm delay which is also $30 \mathrm{~ms}$ (Fig. 9a). So the PMU processing delay is 60 ms.

IEEE C37.118.2 Frame Formation Delay: In order to investigate the delay associated with the packaging of synchrophasors in IEEE C37.118.2 format, the real-time simulator (Opal-RT) [11] was synchronized to a Coordinated Universal Time (UTC) traceable time-source similar to the one provided by GPS. For this purpose, hardware GPS synchronization module from Spectracom (Tsync-PCIe express board) was used [26]. This module provides UTC time-stamp within the real-time environment (using dedicated libraries provided by the vendor Opal-RT) and also provides PPS signal to a clock adapter to generate a synchronized clock. The way the driver of this module works is that it reads the integration time-stamp configured in the mathematical model and generates pulse at corresponding frequency which is aligned to PPS of the GPS source. The synchrophasor stream from the PMU is received inside the simulation model using C37.118 data parsing (C37.118 Master block provided by the vendor Opal-RT). This block captures real PMU streams (based on configuration file setup) and reads these synchrophasors directly inside the simulation model. Figure 10a shows the Opal-RT eMEGAsim Real-Time Simulator architecture with time synchronization module inserted while Fig. 10b shows the Simulink model to read GPS synchronized real-time and to parse synchrophasor stream inside the simulation model. The latency was computed to be $45 \mathrm{~ms}$ with a jitter of 2-3 ms. (Fig. 9b).

GOOSE Latency: PMU streams out GOOSE message with a preconfigured "heartbeat" rate. The heartbeat rate chosen for this study was $100 \mathrm{~ms}$. Every $100 \mathrm{~ms}$, the PMU sends to the communication link the GOOSE messages it is configured to publish. However, if there is a state change in the value of the GOOSE message, the PMU sends a burst of repeated GOOSE messages within 4 to $8 \mathrm{~ms}$, only gradually slowing back down to a heartbeat rate of 10 message per second again (Fig. 9c). The maximum delay encountered by the GOOSE message is thus, 4-8 ms.

Islanding Detection Algorithm Delay: The PMU's protection and control processing capability specifies that the PMU updates its calculations/status at a rate of 8 times per power system cycle [13]. At $50 \mathrm{~Hz}$ the relay processes the logic every $2.5 \mathrm{~ms}$. At a reporting rate of 50 frames $/ \mathrm{s}$ or every $20 \mathrm{~ms}$, the protection logic equations are updated every $2.5 \mathrm{~ms}$. The algorithm is executed every $2.5 \mathrm{~ms}$ irrespective of the complexity of the algorithm. Once the algorithm gets too complex (e.g. if it uses too many protection logic equations and variables), the PMU issues a status representing that the PMU execution capability is exceeded.

The only intentional delay which was introduced was the timer of 10 cycles (PCT01PU), to ensure that the islanding detection scheme only operates if the synchrophasor quantities exceed the threshold for more than 10 cycles [20].
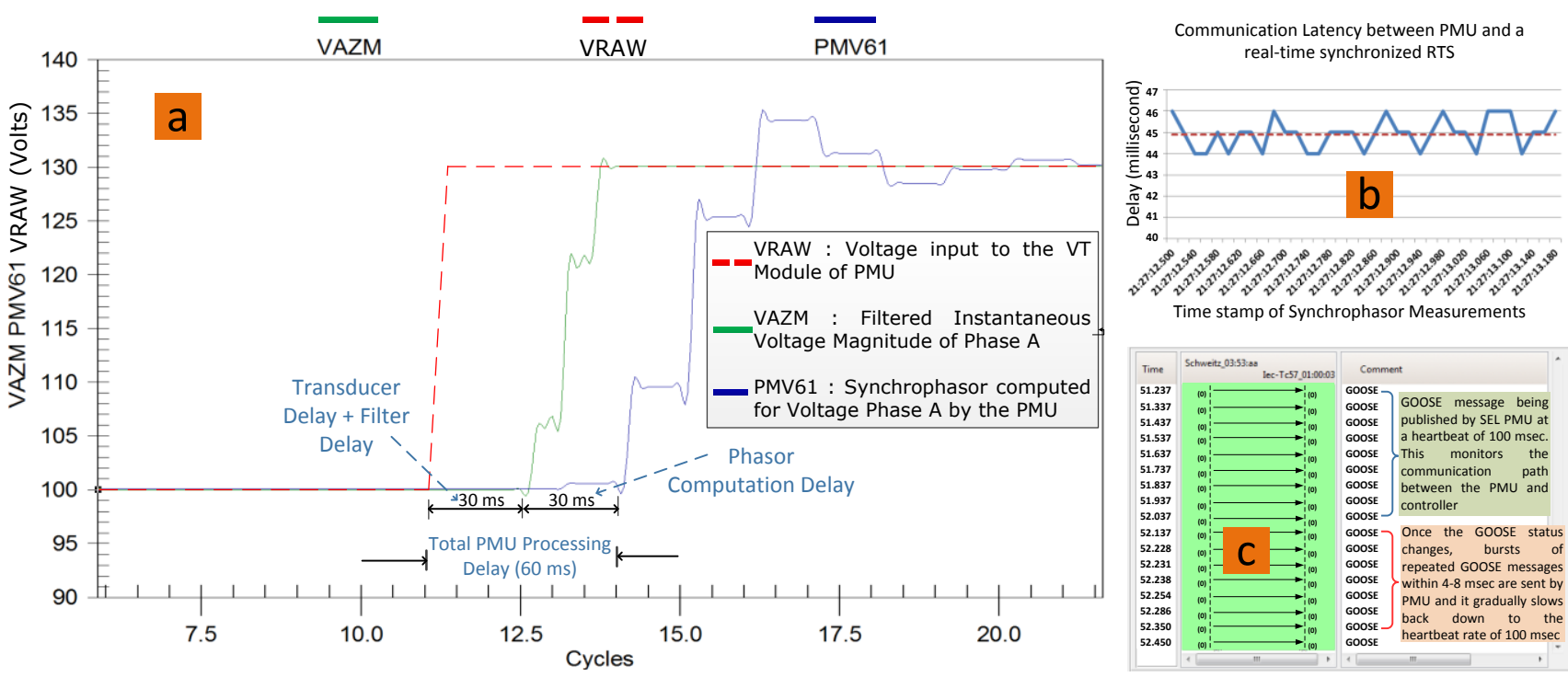

Fig.9. Calculation of different latencies (a) PMU processing delay (60 ms), (b) delay associated in assembling synchrophasors in IEEE C27.118.2 format (45 $\mathrm{ms}$ ), and (c) communication latency of GOOSE messages (4 ms). 

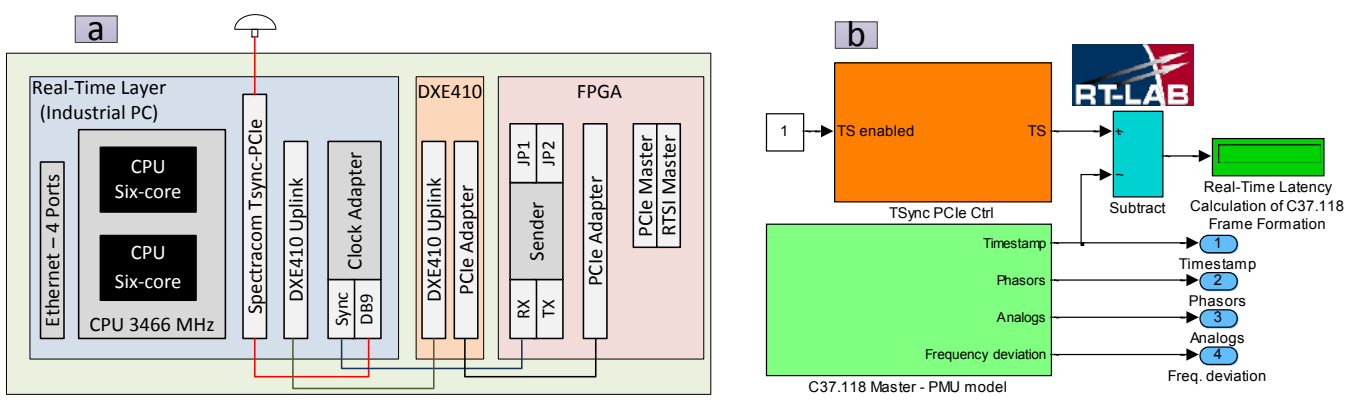

Fig.10. (a) Opal-RT architecture showing the coupling of Spectracom time-synchronization module to synchronize simulator with the GPS, and (b) shows the Simulink model with one block to provide real-time signals while the other block to parse incoming synchrophasor stream from PMU in C37.118.2 format.

\section{WIDE-AREA PASSIVE ISLANDING SCHEMES}

In order to investigate the benefit of utilizing wide-area synchrophasor measurements for the passive islanding algorithms presented in Section-V, the RT-HIL setup shown in Fig. 11a is deployed. PMU-B is considered a local PMU (in the vicinity of a DG) being fed with currents and voltages from Bus-4, while PMU-A is a remote PMU installed at Bus-7 and streaming out synchrophasors at the same rate of 50 frames/s. Experimental sequence presented in Section- $\mathrm{V}$ were repeated to simulate islanding scenario.

The same strategy of deploying islanding detection algorithms within the PMU is carried out by making PMU-B as a client for PMU-A and using direct relay-to-relay communication technique between them. The direct relay-to-relay communication technique allows the two PMUs to exchange synchrophasors directly, without the requirement of an intermediate PDC [13]. This reduces the overall latency of the wide-area synchrophasor based islanding detection schemes, and it further advocates one of the contribution of the paper which is to test and validate these islanding schemes with minimum latencies. Thus, PMU-B processes the remote synchrophasor data, time aligns them with local data internally and makes them available for the passive islanding schemes.

The logic equations used to deploy the over/under voltage based passive islanding detection algorithm using wide-area synchrophasor measurements within PMU-B are shown in Fig.11b. In PMU-B (client), an analog variable is dedicated to store the value of the positive sequence synchrophasor voltage magnitude being received by PMU-A (server). The rest of the algorithm is similar to the one presented in Fig. $3 b$. The only difference being the utilization of the difference in magnitude of positive sequence voltage synchrophasor between local and remote buses to detect islanding conditions. The threshold for this scheme was set to $0.1 \mathrm{pu}$ to compare the results with local synchrophasor-based scheme.

Similarly, wide-area synchrophasor-based islanding detection algorithms using over/under frequency were also implemented. In this case, the absolute value of the difference in synchrophasor frequency between local and remote buses was used to detect islanding. The threshold for this scheme was set to $1 \mathrm{~Hz}$ for comparison purposes.

Finally, a wide-area synchrophasors-based ROCOF islanding detection scheme was implemented by deploying an algorithm in PMU-B that adds absolute value of ROCOF from local and remote PMUs and detects islanding if this value exceeds a threshold of $0.2 \mathrm{~Hz} / \mathrm{s}$.

\section{RT-HIL SIMULATION RESULTS FOR WIDE-AREA PASSIVE ISLANDING SCHEMES}

The NDZ for wide-area passive islanding schemes is shown in Fig. 12, while Fig. 13 and Fig. 14 shows the operating time of these schemes for both active and reactive power mismatch. The operating times of all the schemes decreases with an increase in active or reactive power mismatch between DG and local load (Fig. 13 and Fig. 14).

As compared to NDZ with local synchrophasors (Fig. 5), the NDZ with wide-area synchrophasor for the over/under voltage scheme (Fig. 12a) is reduced from $30 \%$ to $15 \%$, for over/under frequency NDZ is reduced from $10 \%$ to $5 \%$ (Fig. $12 \mathrm{~b}$ ) and for ROCOF-based scheme, the NDZ is reduced from $10 \%$ to $3 \%$ (Fig. $12 \mathrm{c}$ ).

The ROCOF-based scheme results in a faster operation for both active and reactive power mismatches (Fig. 13 and Fig. 14) than the rest of the methods. However, the operating time of ROCOF-based scheme reduces from $0.6 \mathrm{~s}$ (Fig. 7 and Fig. 8) with local synchrophasors to $0.25 \mathrm{~s}$ (Fig. 13 and Fig. 14) with wide-area synchrophasors.

The acceptable operation time of islanding detection scheme with over/under frequency thresholds is achieved when there is an active power mismatch of at least $20 \%$ (Fig. 13) while utilizing wide-area measurements. Whereas an active power mismatch of at least $30 \%$ is required when utilizing local synchrophasors (Fig. 7). Similarly, all the passive islanding detection schemes utilizing wide-area measurements (Fig. 13 and Fig. 14) require less active and reactive power mismatch as compared to local synchrophasor-based schemes (Fig. 7 and Fig. 8) to operate within $2 \mathrm{~s}$.

The wide-area synchrophasor-based islanding detection schemes deployed and analyzed in this section are subjected to one additional delay (in addition to those discussed in SectionVI). This additional delay is $40 \mathrm{~ms}$. It occurs inside PMU-B for synchrophasor acquisition from PMU-A (remote) and its time alignment with local synchropahsors, which is required to execute wide-area islanding algorithms. This latency is calculated by taking the difference of the Fraction of Second [18] associated with synchrophasor frame from PMU-A (remote) and the Fraction of Second of the delayed, time aligned PMUB (local) measurements as shown in Fig. 15. 

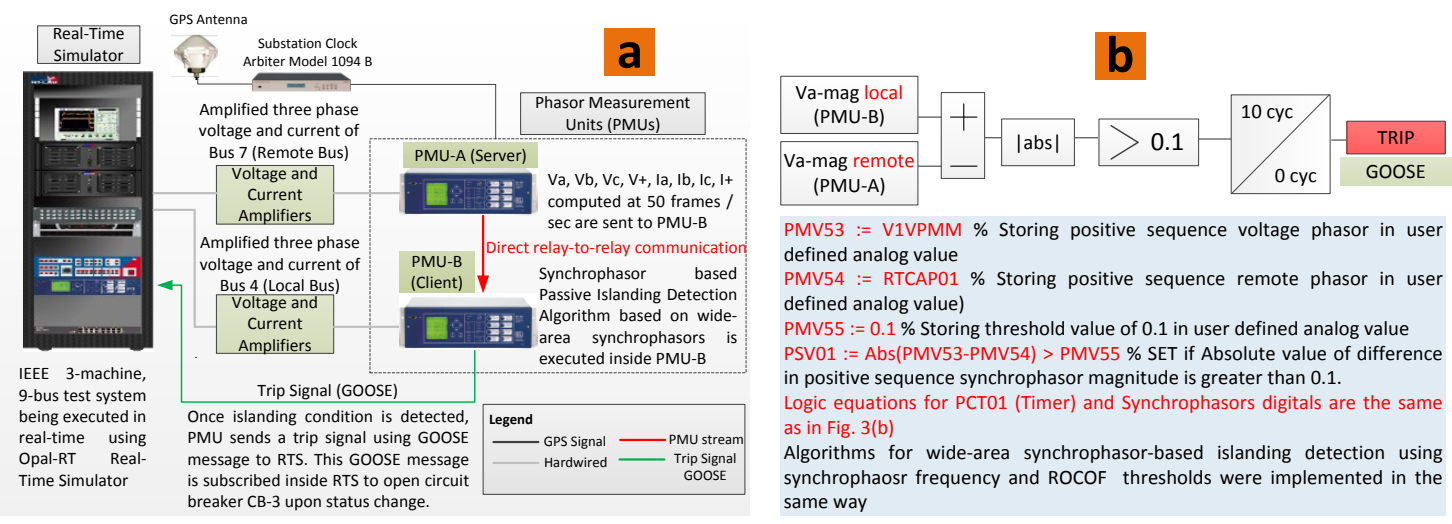

Fig.11. (a) Experimental setup for performance analysis of synchcrophasor based islanding detection schemes using wide-area synchrophasors, and (b) logic equations used to deploy wide-area synchrophasors-based passive islanding scheme using over/under voltage thresholds. Algorithm is deployed within PMU-B

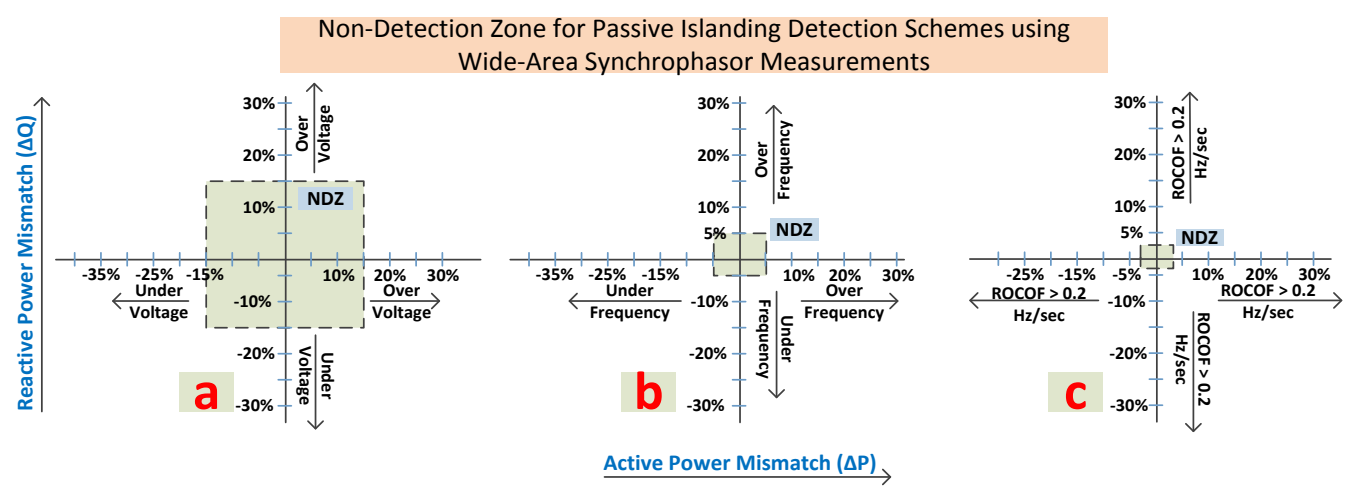

Fig.12. Non Detection Zone (NDZ) for wide-area synchrophasors-based passive islanding detection (a) Under/Over Voltage based islanding detection scheme with abs $\left|V_{B-P M U}-V_{A-P M U}\right| \geq 0.1 \mathrm{pu}$, (b) Under/Over Frequency based detection method with abs $\left|f_{B-P M U}-f_{A-P M U}\right| \geq 1 \mathrm{~Hz}$, and (c) ROCOF based islanding detection scheme with $\mathrm{ROCOF}_{\mathrm{B}-\mathrm{PMU}}+\mathrm{ROCOF}_{\mathrm{A}-\mathrm{PMU}}$ threshold limit set to $0.2 \mathrm{~Hz} / \mathrm{s}$

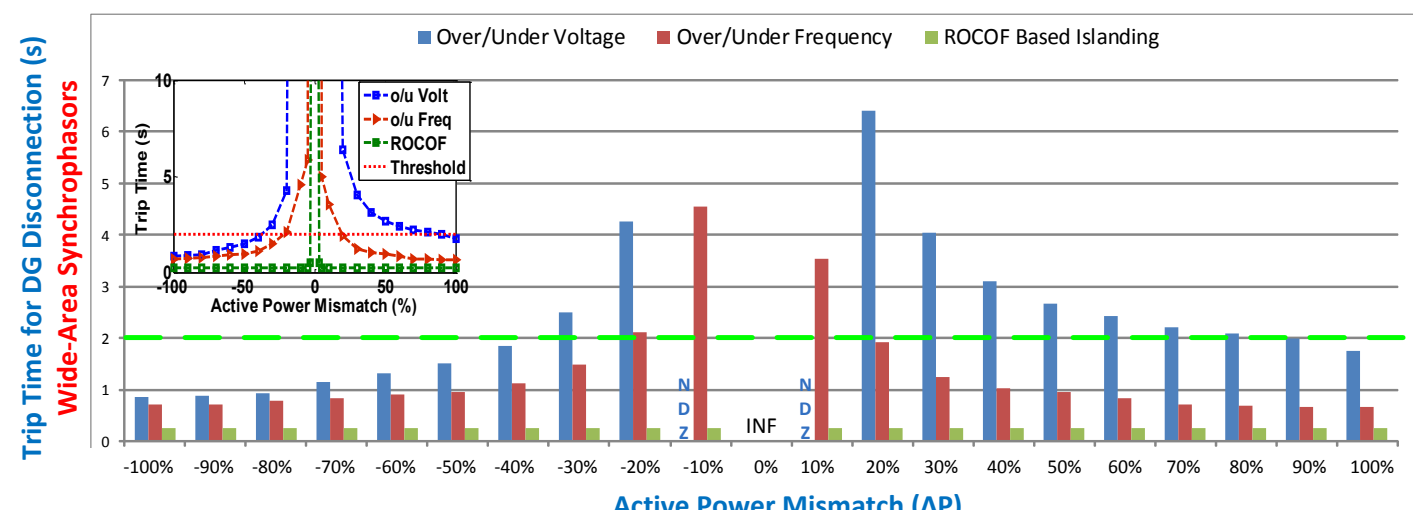

Fig.13. Operation time of passive islanding detection schemes (wide-area synchrophasors) when there is an active power mismatch.

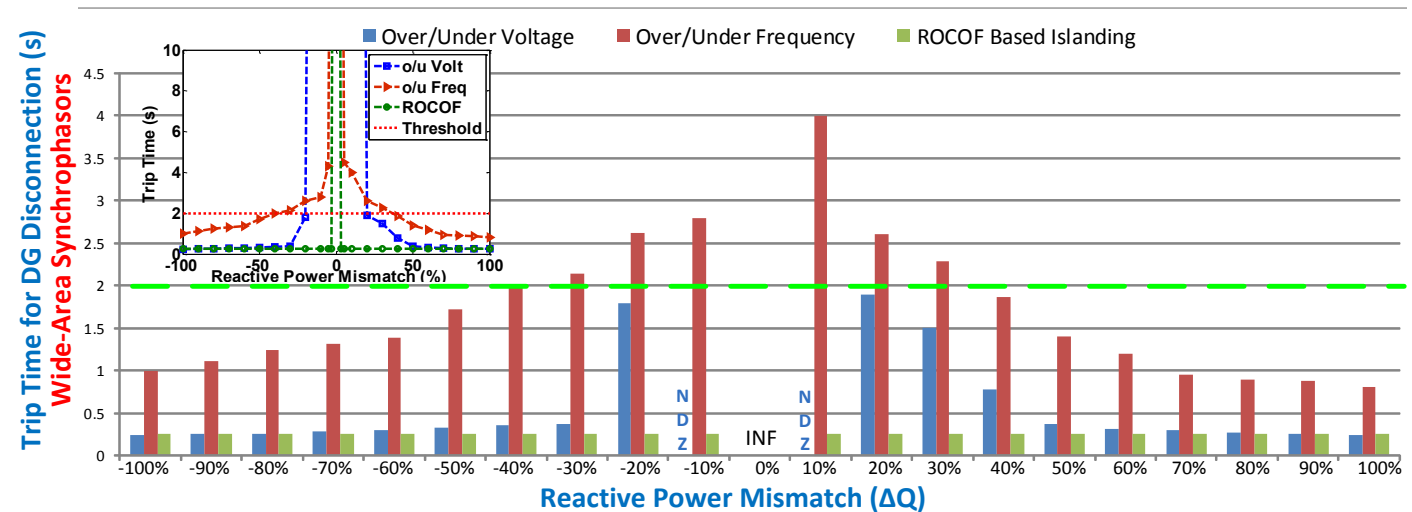

Fig.14. Comparison of operation time of passive islanding detection schemes (wide-area synchrophasors) when there is reactive power mismatch 


\section{CONCLUSION}

This paper presented the implementation and RT-HIL performance assessment of three passive islanding detection methods that exploit local and wide-area synchrophasor measurements and initiate tripping using IEC 61850-8-1 GOOSE messages. ROCOF-based islanding detection schemes are effective for both active and reactive power mismatch, and result in faster operation time as compared to over/under frequency and over/under voltage-based islanding detection schemes. For the same islanding detection techniques, wide-area measurements not only perform faster, but also have smaller NDZs as compared to local synchrophasor-based schemes.

By performing more than 400 RT-HIL experiments, the paper shows that if latencies are kept to a minimum, wide-area passive islanding detection schemes reduce the NDZ to half or two-third of the one using local synchrophasors.

The proposed hybrid schemes ensure minimum communication delays. This is due to the use of synchrophasor measurements internally in a PMU to perform these protection actions using logic equations avoids the delays incurred due to intermediate PDC or IEEE C37.118.2 protocol parser. The

\section{REFERENCES}

[1] F. Katiraei, M. R. Iravani, and P. F. W. P. Lehn, "Micro-grid autonomous operation during and subsequent to islanding process", IEEE Trans. on Power Delivery, vol. 20, No. 1, pp.248 -257, 2005

[2] IEEE Application Guide for IEEE Std 1547, IEEE Standard for Interconnecting Distributed Resources with Electric Power Systems, IEEE Std 1547.2-2008, 2009.

[3] J. De La Ree, V. Centeno, J. S Thorp, and A. G. Phadke, "Synchronized Phasor Measurement Applications in Power Systems", IEEE Trans. on Smart Grid, vol. 1, No. 1, pp.20 -27, 2010

[4] IEC Standard, "Communication networks and systems in substations Part 8-1: Specific Communication Service Mapping (SCSM) - Mappings to MMS (ISO 9506-1 and ISO 9506-2) and to ISO/IEC 8802-3."

[5] H.R. Antti, R. Olli, and S. Janne, "Utilizing possibilities of IEC 61850 and GOOSE", 20th International Conference and Exhibition on Electricity Distribution - Part 1, CIRED, Prague, Czech Republic, June 8$11,2009$.

[6] M.S. Almas and Luigi Vanfretti, "Methodologies for Power Protection Relay Testing-Conventional to RT-HIL Approaches", IPST 2013, Vancouver, British Columbia, Canada, July 2013, available at: http://ipstconf.org/papers/Proc_IPST2013/13IPST002.pdf

[7] R. Franco, C. Sena, G. N. Taranto, and A. Giusto, "Using synchrophasors for controlled islanding - A prospective application for the Uruguayan power system", IEEE Trans. Power Systems, vol. 28, no. 2, pp. 2016-2024, May 2013.

[8] David M. Laverty, Robert J. Best, and D. John Morrow, "Loss-ofmains protection system by application of phasor measurement unit technology with experimentally assessed threshold settings", IET Generation, T \& D, Vol. 9 , No. 2, pp. 146-153, Jan. 2015

[9] J. Guo, et.al, "Design and Implementation of a Real-Time Off-Grid Operation Detection Tool from a Wide-Area Measurements Perspective", IEEE Trans. Smart Grids, vol. 6, no. 4, pp. 2080-2087, Sept. 2014

[10] P. W. Sauer and M. A. Pai, Power System Dynamics and Stability, 1998 :Prentice-Hall

[11] Opal-RT, "eMEGAsim PowerGrid Real-Time Digital Hardware in the Loop Simulator", Available online: http://www.opal-rt.com/.

[12] Y. Liu, M. Steurer, and P. Ribeiro, "A novel approach to power quality assessment: real time hardware-in-the-loop test bed", IEEE Trans. on Power Delivery, vol. 20, No. 2, pp. 1200-1201, April 2005

[13] SEL, "Protection Relays by Schweitzer Engineering Laboratories," available on-line: http://www.selinc.com/protection/.
RT-HIL test-bench proved effective in accurately calculating the latencies such as PMU filtering delay, PMU synchrophasor computation delay, latencies associated with remote measurements time- alignment, PMU algorithm execution delay, synchrophasor frame formation delay and GOOSE message delays.

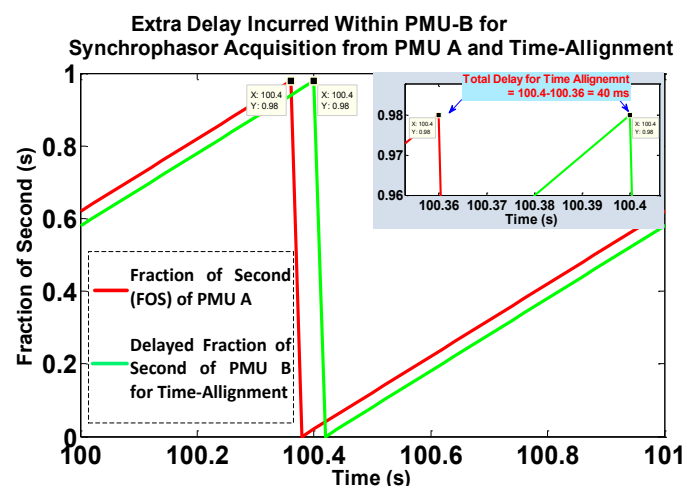

Fig.15. Additional delay of $40 \mathrm{~ms}$ incurred inside PMU-B for synchrophasor acquisition from PMU-A and its time alignment while executing wide-area synchropahsors-based passive islanding detection schemes.

[14] J.C.M Vieira, W. Freitas, X. Wilsun Xu, and A. Morelato, "An Investigation on the Nondetection Zones of Synchronous Distributed Generation Anti-Islanding Protection", IEEE Trans. on Power Delivery, vol. 23, no. 2, pp.593 -600, April 2008

[15] H. Laaksonen, "Advanced Islanding Detection Functionality for Future Electricity Distribution Networks", IEEE Trans. on Power Delivery, vol. 28, no. 4, pp.2056 -2064, Oct. 2013

[16] L. A. C. Lopes and H. Sun, "Performance assessment of active frequency drifting islanding detection methods," IEEE Trans. Energy Conversion, vol. 21, no.1, pp. 171-180, Mar. 2006.

[17] M.S. Almas, M. Baudette, L. Vanfretti, S. Løvlund and J.O. Gjerde, "Synchrophasor Network, Laboratory and Software Applications Developed in the STRONg2rid Project", IEEE PES GM 2014, Washington DC, USA

[18] IEEE Standard for Synchrophasor Data Transfer for Power Systems, IEEE Std C37.118.2-2011, Dec. 2011

[19] L. Vanfretti, V. H. Aarstrand, M. S. Almas, S. P. Vedran, and J. O. Gjerde , "A Software Development Toolkit for Real-Time Synchrophasor Applications", Powertech 2013, Grenoble, France, June 2013

[20] S. I. Jang, and K. H. Kim, "An islanding detection method for distributed generations using voltage unbalance and total harmonic distortion of current," IEEE Tran. Power Delivery, vol. 19, no. 2, pp. 745-752, April 2004.

[21] Vieira, J.C., et al., "Performance of frequency relays for distributed generation protection", IEEE Trans. on Power Delivery, vol. 21, no. 3, pp. 1120-1127, 2006.

[22] M. A. Redfern, O. Usta, and G. Fielding, "Protection against loss of utility grid supply for a dispersed storage and generation unit", IEEE Trans. Power Delivery, vol. 8, no. 3, pp. 948-954, July 1993

[23] M. S. Almas, J. Kilter and L. Vanfretti, "Experiences with Steady-State PMU Compliance Testing using Standard Relay Testing Equipment", IEEE PQ Conference, Estonia, 2014

[24] J. Kilter, M. S. Almas, I. Palu and L. Vanfretti, "Experiences with PMU Dynamic Compliance Testing using Standard Relay Testing Equipment", Innovative Smart Grid Conference (ISGT) North America, Washington DC, USA, Feb 2015

[25] P. Romano, and M. Paolone, "Enhanced Interpolated-DFT for Synchrophasor Estimation in FPGAs: Theory, Implementation, and Validation of a PMU Prototype", IEEE Transactions on Instrumentation and Measurement, vol. 63, no. 12, pp. 2824-2836, May 2014

[26] Spectracom PCI Express Slot Card [online]. Website: http://spectracom.com/ProductsServices/TimingSynchronization/BuslevelTiming/PClexpressslotcards/tabid/1296/Default.asp 